A. Herzen: Ueber d. Einfluss d. Milz auf d. Bildung d. Trypsins. 295

\title{
Ueber den Einfluss der Milz auf die Bildung des Trypsins.
}

\author{
Von
}

\section{Prof. Dr. Alexander Herzen}

in Lausanne.

Schon $1862^{\mathrm{I}}$ ) veröffentlichte $M$. Schiff eine lange Versuchsreihe, die zu dem Resultate führte, dass nach Ausrottung der Milz der Pankreassaft oder das Infus der Drïse ihre eiweissverdauenden Eigenschaften auf immer einbüssen; fernere Untersuchungen führten ihn zum Schlusse, dass die Milz nicht direct zur Bildung des Trypsins beitrage, sondern während ihrer functionellen Dilatation ein Ferment bilde, welches für die im Blute gegenwärtigen Peptogene eine nothwendige Bedingung ihrer Umwandlung in Pankreatin sei.

1875 veröffentlichte Heidenhain seine bekannten Untersuchungen über die Gegenwart und Bildung des Zymogens im Pankreas. Seine Ergebnisse schienen mit denjenigen Schiff's in Widerspruch zu stehen, denn das Zymogen häuft sich, ganz unabhängig von der Milz, in den Pankreaszellen an. Bei näherer Ueberlegung fiel es mir auf, dass das Zymogen sich im Pankreas immer in umgekehrtem Verhältniss zu der Menge des gebildeten Trypsins vorfindet; das Minimum des Einen entspricht dem Maximum des Andern, und umgekehrt. Da aber die Menge des gebildeten Trypsins in directem Verhältniss zu der Dilatation der Milz steht, so wurde es noch wahrscheinlicher, dass die Milz eben während ihrer Dilatation das z ymogenumwandelnde Ferment bilde.

Zur experimentellen Prïfung dieser Hypothese machte ich $1877^{2}$ ) folgenden Versuch: Ich tödtete einen seit 24 Stunden nuich-

1) M. Schiff, Schweiz. Zeitschr. f. wissenschaftl. Medicin.

2) A. Herzen, Moleschott's Untersuchungen zur Naturlehre des Menschen und der Thiere. Bd. XII, 1. Heft. 
ternen Hund, und gleichzeitig einen anderen Hund, der sich zwischen der 6. und der 7. Stunde der Verdauung befand. Das blasse Pankreas des nuichternen Hundes wurde in drei Theile getheilt: der erste wurde sogleich in Glycerin infundirt; der zweite wurde mit einem Stücke der Milz desselb en Hundes zerrieben, und dann infundirt; der dritte wurde mit einem Sticke der dilatirten Milz des verdauenden Hundes zerrieben, und sogleich infundirt. In diese drei Infuse legte ich gleiche Mengen frisch gekochten Eiweisses; das Ergebniss war folgendes:

Pankreas des nitchternen Hundes allein - verdaut nichts;

dasselbe mit der Milz desselben Hundes - verdaut nichts; dasselbe mit der Milz des anderen Hundes - verdaut alles.

Auf diese Weise wären Schiff's und Heiden hain's Resultate nicht mehr im Widerspruch, sondern vielmebr in vollkommener Uebereinstimmung mit einander, und als sich gegenseitig ergänzende zu betrachten.

Eventuell habe ich beobachtet, dass die Milz nicht bei jeder Verdau ung anschwillt, und dass, wenn sie trotz der Verdaung klein und anämisch bleibt, das Pankreasinfus kein Eiweiss verdaut. Diese Thatsache ist eine neue Bestätigung der Schiff'schen Resultate, und erklärt wahrscheinlich auch, warum Heidenha in glaubt, dass auch im verdanenden Thiere kein Trypsin im Pankreas vorhanden sei. Man muss doch annehmen, dass dasselbe sich auch im lebenden Pankreas unter gewissen Bedingungen bilde; wozu wäre sonst das Zymogen da, und woher käme das Trypsin im Pankreassafte?

Die physiologische Bedingung für die Umwandlung des Zymogens in Trypsin ist die Bildung des Milzfermentes, welche ibrerseits wiederum durch die functionelle Dilatation der Milz bedingt ist.

In Hermann's Handbuch der Physiologie erwähnt Heidenhain meiner Versuche, und fügt hinzu, dass sie ,sehr der Bestätigung bedürfen". Da ich diese Meinung mit ihm vollständig theilte, so habe ich auch im Verlaufe dieses Sommers eine neue Versuchsreihe nnternommen, die mir trotz einer viel grösseren Zahl Versuche und einer bei weitem strengeren Methode doch genau dieselben Resultate gegeben hat.

Ueber diese letzten Versuche möchte ich nun hier berichten.

Was die Methode betrifft, so mache ich besonders auf folgende Umstände anfmerksam: 
1. Das Glycerin habe ich in meinen Versuchen als Vehikel vermieden, denn obschon es die zwei grossen Vortheile hat, die Fäulniss zu verhindern und die spontane Oxydation des Zymogens bedeutend zu verlangsamen ${ }^{1}$ ), so hat es doch den nicht geringen Nachtheil, auch die Verdauung mehr oder weniger zu verlangsamen, so dass der Gang der Verdaung im Glycerin-Infus uns durchans kein richtiges Bild vom wirklichen Gange der Verdaung giebt.

2. Ieh habe mich ausschliesslich einer 5\%igen Borsäurelösung als Vehikel bedient, weil mir die Erfahrung gezeigt hat, dass sie alle Vortheile des Glycerins besitzt, ohne jedoch den eben erwähnten Nachtheil mit demselben zu theilen. Ja, oft geschieht es, dass die Verdaung in Borsäureinfus raseher vor sich geht als in Wasserinfus.

3. Jede einzelne Verdauungsprobe habe ich immer zugleich mit Faserstoff und Eiweiss gemacht, denn es geschieht öfter als man es wohl denkt, dass dasselbe Infus ziemlich rasch eine mittelmässige Menge Faserstoff peptonisirt, während es das Eiweiss nicht nachweisbar angreift.

4. Sämmtliche Infuse habe ich augenblicklich nach dem Tode des Thieres gemacht, jedes Stïck des infundirten Organs in $10 \mathrm{Mal}$ sein Gewicht Vehikel gebracht, 24 Stunden stehen lassen und dann probirt, bei 37 bis $40^{\circ} \mathrm{C}$. Die Probe hat immer 24 Stunden gedauert; die Beobachtung derselben wurde vier Mal unternommen: nach 1 Stunde, nach 3 Stunden, nach 6 Stunden, nach 24 stunden. Da nun aucb die Menge Faserstoff und Eiweiss, empirisch als die passendste erkannt, immer dieselbe blieb, so habe ich zur Bestimmung der relativen Mengen des verdauten Stoffes vollkommen anf chemische Reaktionen und auf Wiegen der trocknen Residuen verzichtet. Es kommt ja hauptsächlich darauf an zu erkennen, welches der Infuse in den ersten Stunden der Probe am meisten verdaut; und das erkennt man ganz gut, mit etwas Uebung, mit dem blossen Auge für den Faserstoff, und mit einer Lape für das Eiweiss. Bis zur 6 . Stunde hat die Beobachtung ihren vollen Werth; später, je länger die Probe dauert, desto weniger Bedeutung hat sie, wegen der spontanen Oxydation des Zymogens; die Verdauung verläuft in einem an Trypsin reichen Infus sehr rasch in den ersten

1) Concentrirtes Glycerin verhindert sie ganz, aber wenn man es mit 1 oder 2 Vol. Wasser verdünnt, so verlangsamt es sie nur. 
3 Stunden, und wird dann gewöhnlich allmählich langsamer; das Umgekehrte gilt für ein Infus, welehes von Anfang an nur Zymogen enthält: die Verdauung fängt sehr spät an, nnd die Verdauungskurve geht immer steigend und immer steiler, bis endlich beide Kurven, die erste beinahe horizontal, die zweite beinahe senkrecht, zusammentreffen, wenn man die Probe lange genug fortsetzt -24 , 36, 48 Stunden, wobei die zweite gewöhnlich am Ende mehr leistet.

5. Es kam mir gar nicht darauf an, an entmilzten Hunden zu arbeiten, anch war mir die Verdaungsperiode, in der die Thiere, welche das Pankreas-Infus liefern sollten, getödtet wurden, gleichgültig; nur die zwei Hunde, die das Milz-Infus liefern sollten, mussten in voller Verdaung sein; denn meine Versuche batten keinen andern Zweck als eben zu sehen, ob das Gemenge der Pankreasinfuse mit den Milzinfusen $r a s c h e r$ verdane als die Ersteren allein, und dadurch die rasche Bildung des Trypsins verrathen würde.

Nun gebe ich die graphische Darstellung einiger meiner Versuche. Die Zahlen der Abscisse bedeuten die Stunden der Probe; die Zahlen der Ordinate die relativ verdanten Mengen; also $0=$ Nichts; $1=$ Anfang der Verdauung; $5=$ die Hälfte gelöst; $10=$ Alles gelöst.

Mit jedem der 5 Pankreasinfuse mache ich folgende doppelte Proben, mit Faserstoff die eine, mit Eiweiss die andere:

1. Ein constantes Volum Infus mit 2 Vol. ausgekochten, destillirten Wassers.

2. Dasselbe Volum mit 2 Vol. $5 \%$ Borsäure.

3. Dasselbe Volum mit 2 Vol. Borsäure-Milz-A ufguss.

4: Dasselbe Volum mit 2 Vol. Wasser-Milz-Aufguss. 
Faserstoff-Verdaung.

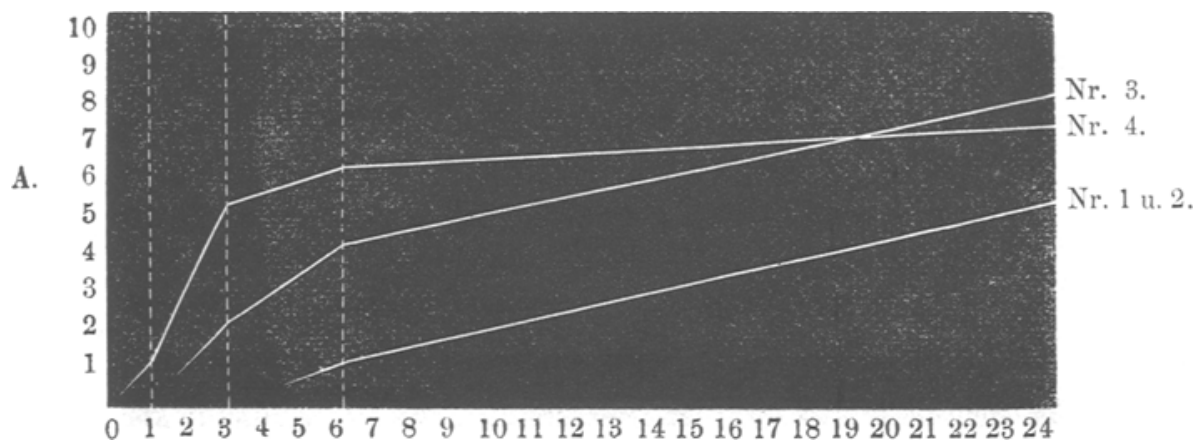

$\mathrm{Nr} .1$ und 2 verlaufen zusammen. Nr. 3 ist offenbar viel wirksamer und Nr. 4 noch mehr, besonders im Anfang.

Eiweis/s-Verdaung.

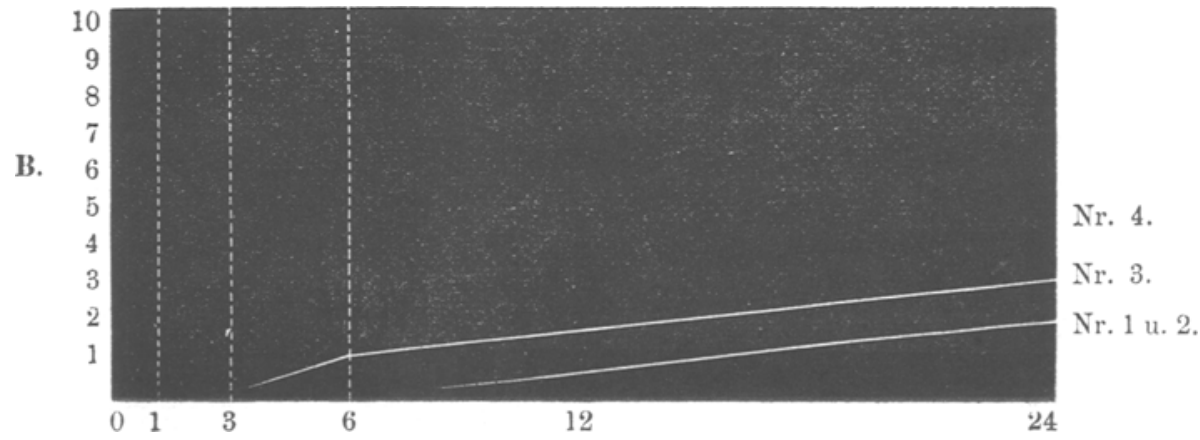

Nr. 1 und 2 verdauen gar kein Eiweiss in 24 Stunden.

Nr. 3 fängt erst in der 12. Stunde an und verdaut nur 2.

Nr. 4 verdant in 6 Stunden 1 und in 24 Stunden 3. 
II.

Faserstoff.

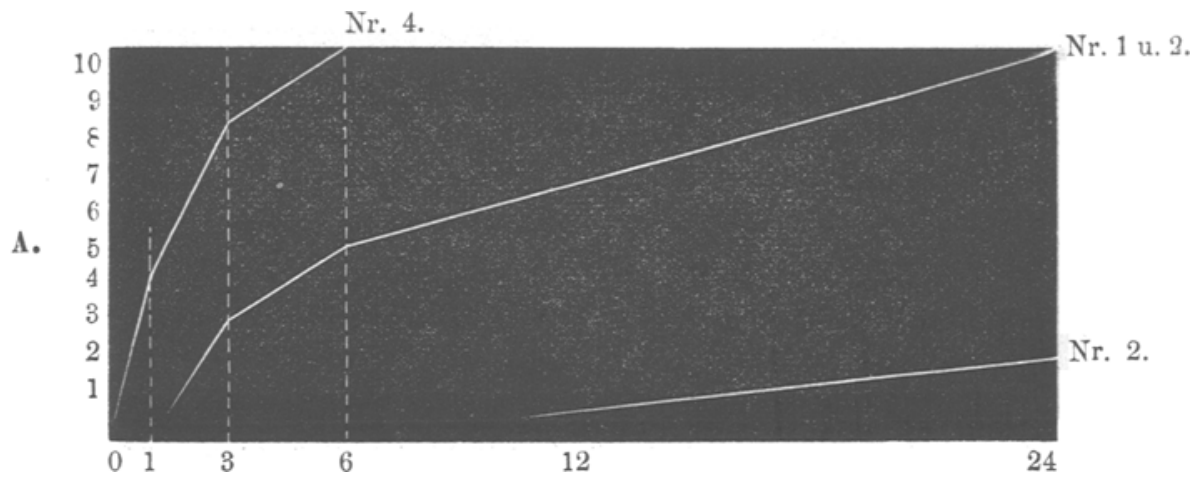

Merkwürdiger Weise verlaufen in diesem Falle Nr. 1 und Nr. 3 zusammen.

Die rasche Verdaung in Nr. 4 ist sehr deutlich.

Nr. 2 leistet ausserordentlich wenig.

\section{Eiweiss.}

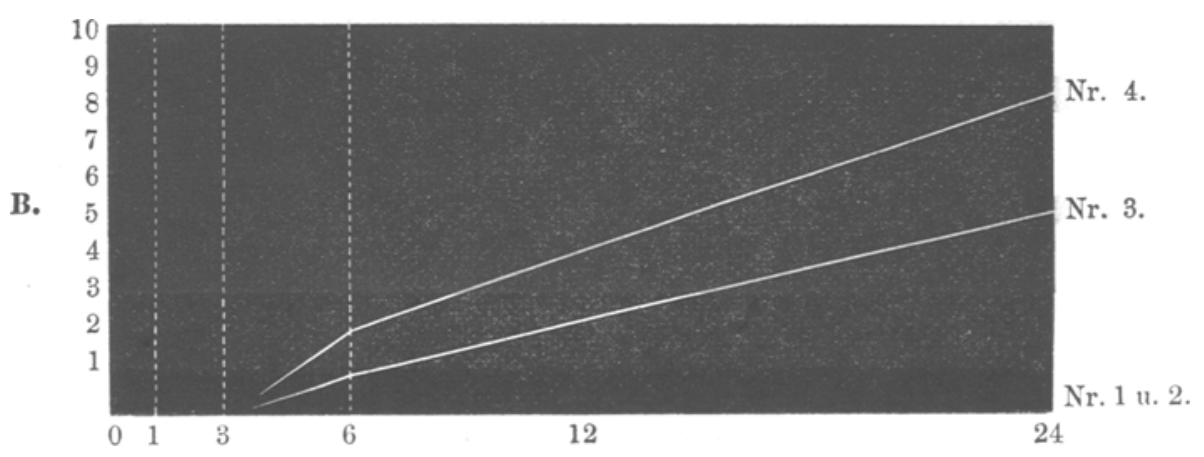

Es wird nur unter dem Einfluss der Milzaufgüsse verdaut. 
Ueber den Einfluss der Milz auf die Bildung des Trypsins.

301

III.

Faserst off.

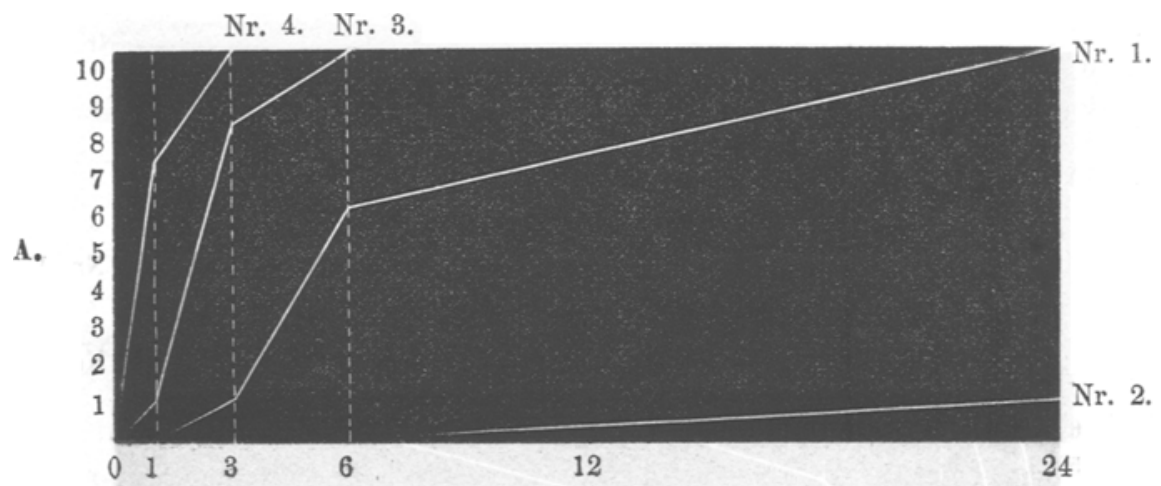

Eiweiss.

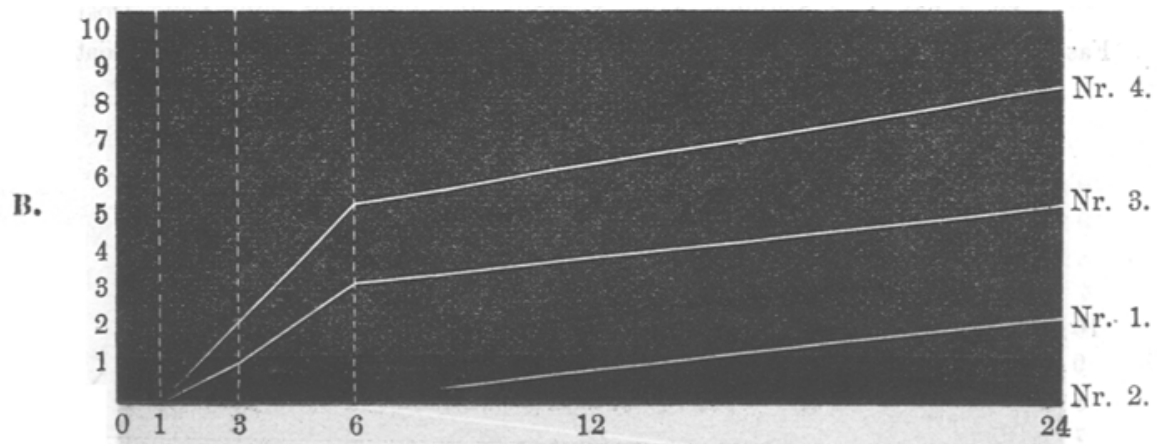


IV.

\section{Faserstoff.}

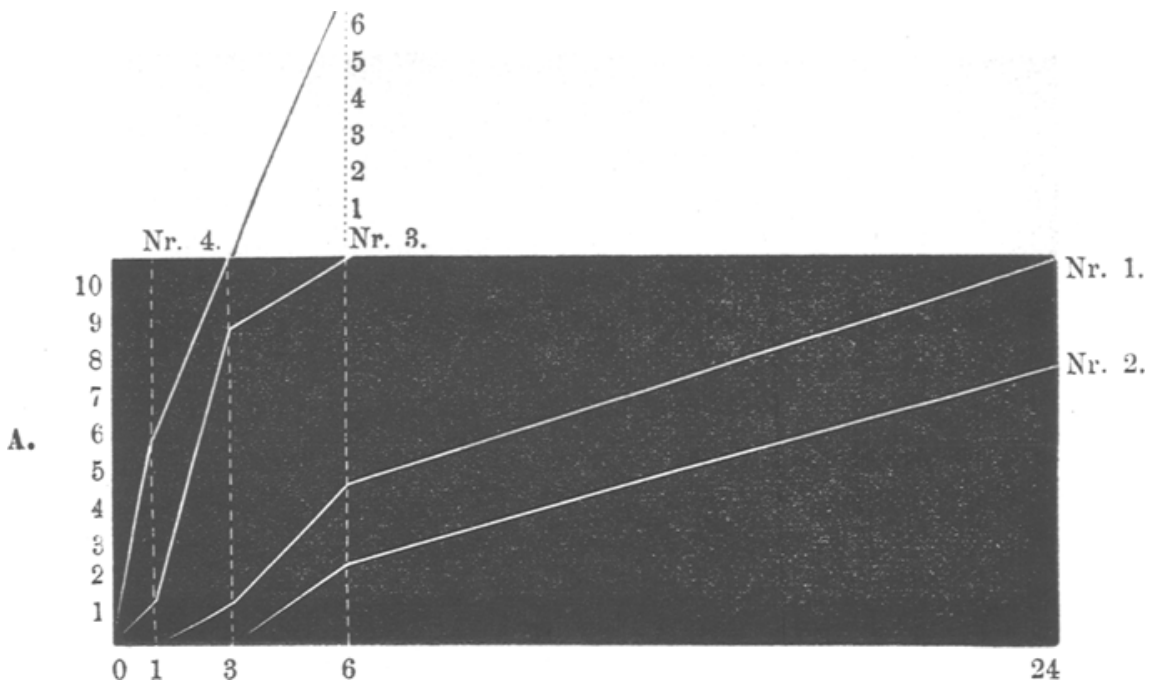

$\mathrm{Nr} .4$ hat in 3 Stunden Alles verdaut und bekommt eine nene Dosis Faserstoff; am Ende der 6. Stunde ist mehr als die Hälfte derselben gelöst.

Eiweiss.

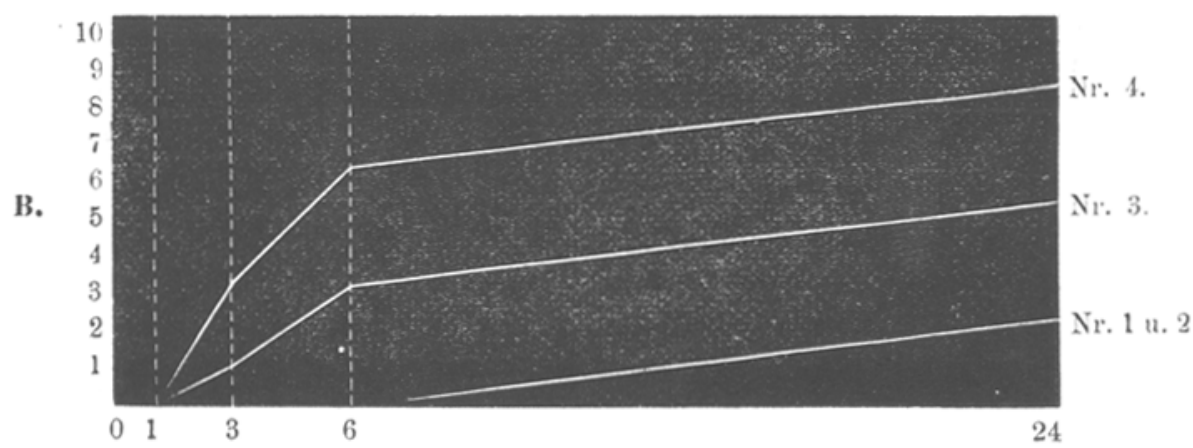




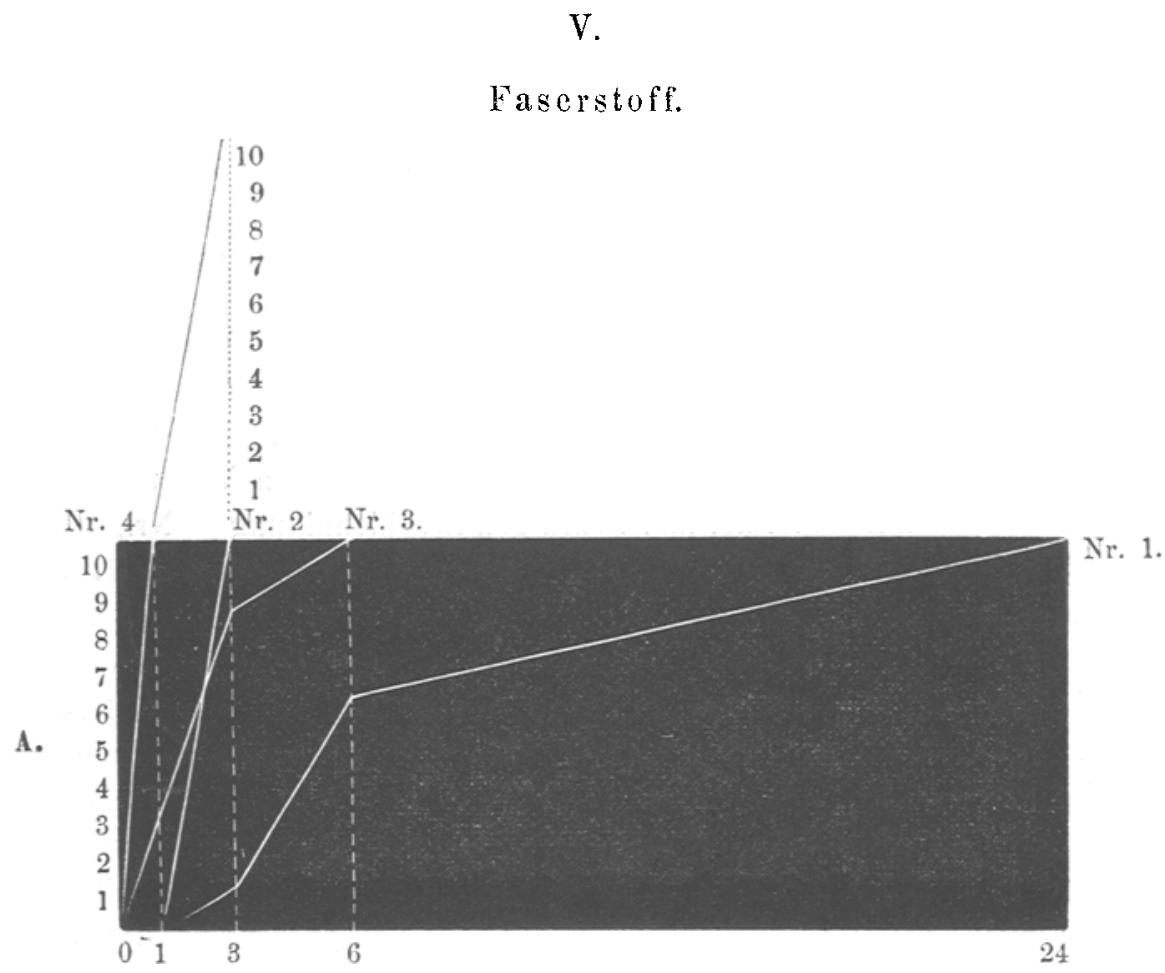

In einer Stunde hat Nr. 4 Alles gelöst, und löst die zweite Dosis am Ende der 3. Stunde. Auffallend ist die ausserordentlich rasche Verdauung in Nr. 2.

Eiweiss.

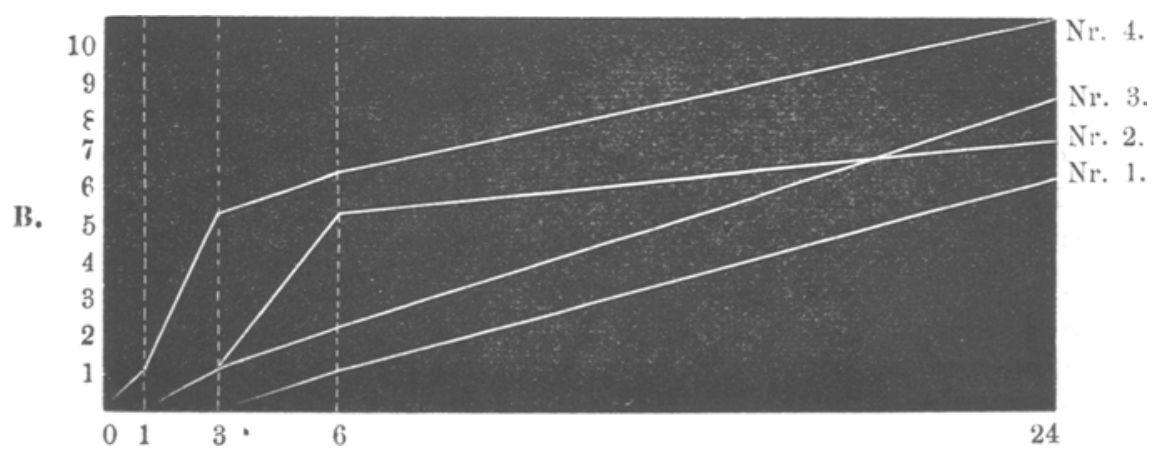

Auch hier ist eine bedeutende Beschleunigung in Nr. 2 deutlich. Zum ersten Male sieht man in diesem Versuche das ga $\mathrm{nz}$ e Eiweiss verschwinden - und zwar unter dem Einfluss des Wasser-Milzaufgusses. 
Wenn wir das Resultat der angeführten Beispiele summiren, so erhellt Folgendes daraus:

In allen Versuchen hat $\mathrm{Nr}$. 4 am raschesten und am meisten verdaut; nur in $1 \mathrm{~A}$ sieht man gegen Ende der Probe eine kleine Unregelmässigkeit.

Nr. 3 zeigt sich in seinem Verdauungsvermögen dem Nr. 4 am ähnlichsten, ist aber offenbar schwächer.

Nr. 1 und 2 sind beide noch viel träger und schwächer als Nr. 3. Sie verlaufen meistens zusammen oder parallel; nur in II A verläuft $\mathrm{Nr} .1$ zusammen mit $\mathrm{Nr}$. 3, nicht aber in II B. In III $A$ und B zeigt sich Nr. 1 thätiger als Nr. 2. In V A und B haben wir ein Beispiel der merkwürdigen Beschleunigung in Nr. ${ }^{1}$ ).

Warum ist nun $\cdot \mathrm{Nr}, 4$ in allen Versuchen am thätigsten? Die Pankreasinfuse reagiren wegen des angewendeten Vehikels schwach saner; sollte die Erscheinung von ihrer Neutralisation durch den Wasser-Milz-Aufguss abhängen? Nein, denn erstens hat ja der Borsäure-Milz-Aufguss ebenfalls eine beschleunigende Wirkung auf Trypsinbildung: in den Eiweiss-Proben ganz ausnahmslos; zweitens sei es mir erlaubt einen Controll-Versuch mit Glycerin-Infusen anzuführen. In der folgenden Tafel ist $\mathrm{Nr} .1$ ein Glycerin-Infus eines Theiles desselben Pankreas, welches den Borsäure-Aufguss für Tafel V geliefert hat mit 2 Vol. ausgekochten distillirten Wassers verdiinnt, und Nr. 2 ein Gemisch von Nr. 1 mit gleichem Volum eines Glycerin-Infuses eines Theiles derselben Milz, die für alle übrigen Versuche den Wasser-Aufguss geliefert hat, mit 4 Vol. ausgekochten distillirten Wassers; $A=$ Faserstoff, $B=$ Eiweiss.

1) Diese Erscheinung bitte ich in der vorliegenden Mittheilung ausser Betracht zu lassen; sie hat eigentlich mit dem Zweck meiner Versuche nichts zu thun. Thatsache ist es, dass ein gewisser Gehalt des Infuses an Borsäure die Peptonisirung oft mehr oder weniger beschleunigt, und manchmal verlangsamt. Ich weiss mir die Sache nicht zu erklären, nur weiss ich, dass sie bei Pepsin-Verdauungen viel häufiger erscheint als bei Trypsin-Verdauungen, ohne dass die Borsäure als Säure dabei eine Rolle spiele, denn ein BorsäureMagen-Aufguss verdaut absolut nichts, so lange man ihn nicht mit HCl ansäuert. 


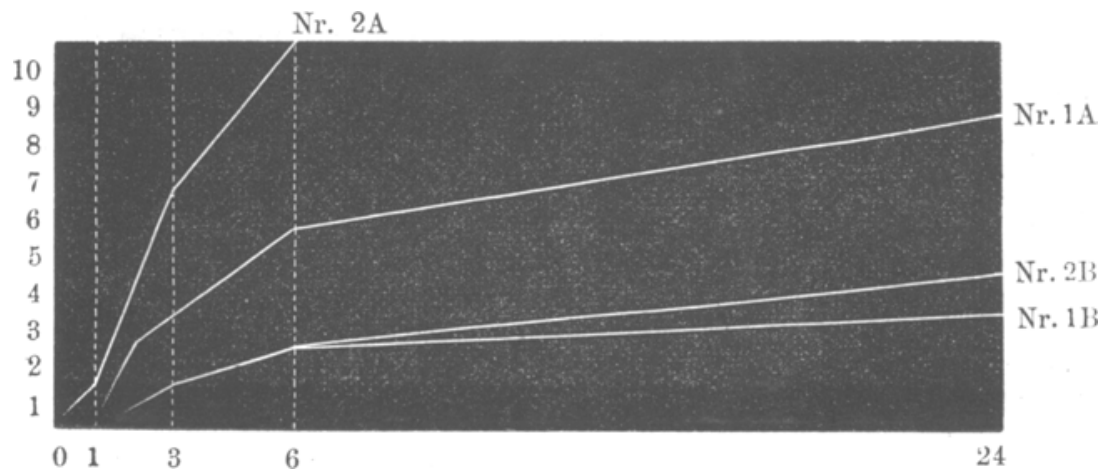

Der Unterschied zwischen $1 \mathrm{~B}$ und $2 \mathrm{~B}$ ist gering, aber zwischen $1 \mathrm{~A}$ und $2 \mathrm{~A}$ ist er eben so ausgesprochen als bei den Borsäure- und Wasser-Aufgüssen.

Wenn also weder die Neutralisation noch die Gegenwart der Borsäure die Beschleunigung der Verdauung erklären, so kann dieselbe nur von der Gegenwart eines unbekannten, in dem MilzInfuse enthaltenen Stoffes abhängen. Um diesen Punkt ganz ausser Zweifel zu setzen, habe ich noch einige Versuche gemacht, indem ich die Pankreas-Infuse mit einem Leber- oder Nieren-Aufguss vermischte, und niemals habe ich eine vermehrte, ja gewöhnlich eine verminderte Verdaung von solchen Gemengen bekommen.

Nun fragt es sich, warum ist in allen Versuchen Nr. 4 thätiger als Nr. 3? Bei der Eröffnung der zwei Milzhunde war es ganz evident, dass nur einer derselben sich wirklich auf dem Höhepunkte der Verdanung befand und eine maximale Congestion der Milz hatte; gerade diese Milz benutzte ich zum Wasser-Aufgusse; glïcklicher Weise infundirte ich aber ein kleines Stück derselben in Borsäure, zu einem eventuellen Controllversuch, über dessen Resultat folgende Tafel berichtet:

Nr. 1 ist der Borsäure-Milz-Aufguss der für alle angeführten Beispiele gebraucht wurde;

Nr. 2 ist der Borsäure-Aufguss von derjenigen Milz, die für alle Versuche den Wasser-Aufguss gab;

Alle drei sind mit dem Borsäure-Infus des Pankreas II gemischt. $A=$ Faserstoff, $B=$ Eiweiss. 


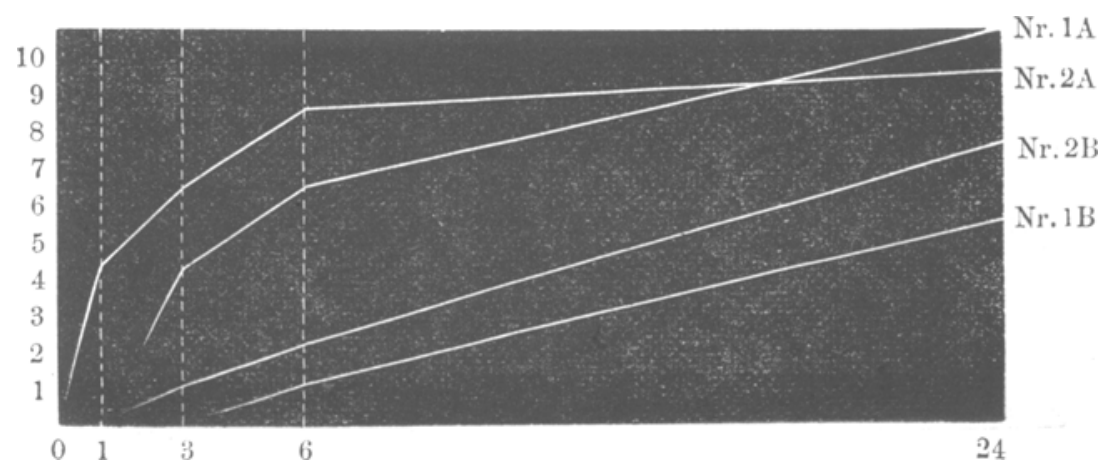

Darnit scheint es mir genügend bewiesen, dass sich in meinen Versuchen der Wasser-Milz-Aufguss thätiger erwiesen hat, nicht weil er eben ein Wasser-Aufguss war, sondern weil die Milz, die ihn lieferte, thätiger war als die andere, die ich zum BorsäureAufguss benutzte.

Beiläufig sei bemerkt, dass ich durchaus nicht behaupte, dass eine geschrumpfte und anämische Milz ganz unthätig sei; es scheint mir im Gegentheil, dass eine solche dieselbe Wirkung auf die Pankreas-Infuse hat, nur viel weniger als wenn sie dilatirt ist.

In meinen sämmtlichen Versuchen, die ca. 20 sind, also ca. 80 Verdauungsproben, sind mir nur zwei Ausnahmen vorgekommen, nämlich solche Proben, wo das Gemenge des Pankreas-Infuses mit dem Milz-Aufguss etw as weniger verdaute als ersteres allein. Diese Ausnahmen erkläre ich mir dadurch, dass in beiden Fällen das ganze vorhandene Zymogen schon in Trypsin verwandelt war, und ein Theil des letzteren dazn gebrancht wurde erst die im Milzaufgusse gegenwärtigen gelösten Eiweisskörper zu peptonisiren, und folglich weniger Faserstoff und Eiweiss peptonisiren konnte. Auch diese Vermuthung bitte ich um Erlaubniss durch folgenden Controllversuch zu documentiren :

Nr. 1 ist ein frisch bereiteter Glycerin-Pankreas-Aufguss.

Nr. 2 derselbe mit Glycerin-Milz-Aufguss.

Nr. 3 derselbe allein, aber einige Wochen später probirt, nachdem sämmtliches, in sehr geringer Menge vorhandene 
Zymogen sich durch direkte Oxydation in Typsin umgewandelt hatte ${ }^{1}$ ).

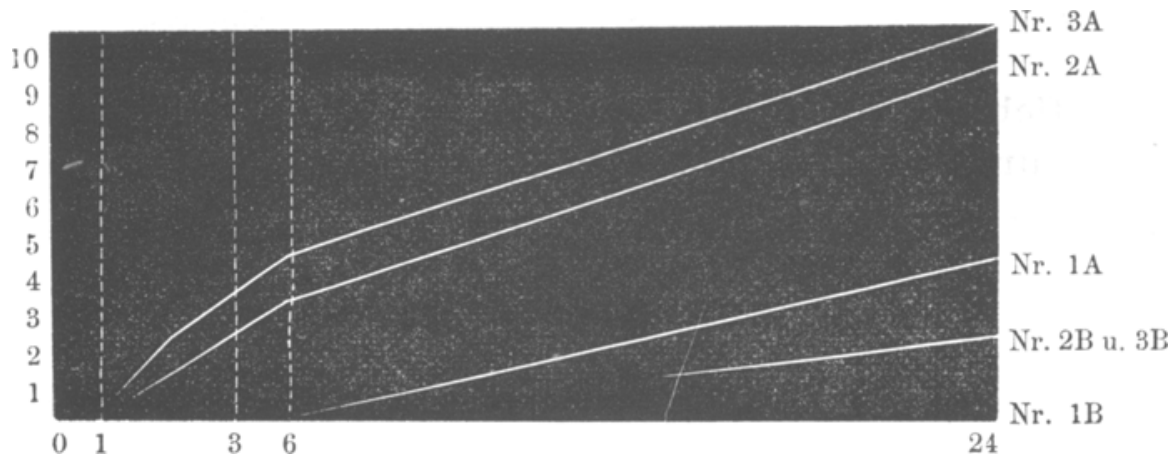

Schluss.

Das im Pankreas gebildete Zymogen wird durch ein in der Milz, während ihrer periodischen Dilatation, gebildetes Ferment zu Trypsin umgewandelt.

1) Der Hund warde 24 Stunden, nachdem er eine kolossale Menge Knochen und Sehnen gefressen hatte, getödtet; es war also in seinem Pankreas der Vorrath an Zymogen beinahe ganz erschöpft. 